Journal of Computer Science 5 (3): 233-241, 2009

ISSN 1549-3636

(C) 2009 Science Publications

\title{
Optimizing Inventory Using Genetic Algorithm for Efficient Supply Chain Management
}

\author{
${ }^{1}$ P. Radhakrishnan, ${ }^{2}$ V.M. Prasad and ${ }^{3}$ M.R. Gopalan \\ ${ }^{1}$ Department of Computer Science and Engineering, \\ PSG Institute of Advance Studies, Coimbatore-641004, Tamil Nadu, India \\ ${ }^{2}$ Jawaharlal Nehru Technological University, School of Management Studies, \\ Hyderabad-500 072 Andhra Pradesh, India \\ ${ }^{3}$ The Institute of Finance and International Management Business School, \\ Bangalore-560100, Karnataka, India
}

\begin{abstract}
Problem statement: Today, inventory management is considered to be an important field in Supply chain management. Once the efficient and effective management of inventory is carried out throughout the supply chain, service provided to the customer ultimately gets enhanced. Hence, to ensure minimal cost for the supply chain, the determination of the level of inventory to be held at various levels in a supply chain is unavoidable. Minimizing the total supply chain cost refers to the reduction of holding and shortage cost in the entire supply chain. Efficient inventory management is a complex process which entails the management of the inventory in the whole supply chain and getting the final solution as an optimal one. In other words, during the process of supply chain management, the stock level at each member of the supply chain should account to minimum total supply chain cost. The dynamic nature of the excess stock level and shortage level over all the periods is a serious issue when implementation was considered. In addition, consideration of multiple products leads to very complex inventory management process. The complexity of the problem increases when more distribution centers and agents were involved. Approach: In present research, the issues of inventory management had been focused and a novel approach based on genetic algorithm had been proposed in which the most probable excess stock level and shortage level required for inventory optimization in the supply chain is distinctively determined so as to achieve minimum total supply chain cost. Results: The analysis provided us with an inventory level that made a remarkable contribution towards the increase of supply chain cost. We predicted the optimal inventory levels in all the supply chain members with the aid of these levels. Conclusion: We concluded that it is possible to minimize the supply chain cost by maintaining the optimal stock levels that we predicted from the inventory analysis. This will make the inventory management further effective and efficient thereby enhancing the customer servicing levels.
\end{abstract}

Key words: Supply chain management, inventory control, inventory optimization, genetic algorithm, supply chain cost

\section{INTRODUCTION}

Global competition, shorter product life cycles, dynamic changes of demand patterns and product varieties and environmental standards cause remarkable changes in the market scenario thereby thrusting the manufacturing enterprises to deliver their best in order to strive $^{[1]}$. Decrease in lead times and expenses, enrichment of customer service levels and advanced product quality are the characteristics that determine the competitiveness of a company in the contemporary market place ${ }^{[11]}$. The above mentioned factors have made the business enterprises to contemplate about their supply chains. An ensemble or organizations providing products and services to the market may be called as a supply chain. A supply chain can also be described as a collection of numerous entities that work in unison towards (1) Obtaining raw materials, (2) Converting these raw materials into precise end products and (3) Delivering the end products to retailers ${ }^{[13]}$.

The activities included in a traditional supply chain are purchase of raw materials and manufacturing items

Corresponding Author: P. Radhakrishnan, Department of Computer Science and Engineering, PSG Institute of Advance Studies, Coimbatore-641004, Tamil Nadu, India 
at one or more factories, shipping the items to various warehouses for storage and in turn distribution of the same to the respective retailers or customers ${ }^{[14]}$. Hence, a beneficial coordination and integration of organizations with individual objectives to achieve a common goal can be referred to as a supply chain.

Processes that support the effective integration of suppliers, manufacturers, warehouses and stores to guarantee suitable production and distribution of right quantities to the right location in right time and decreasing the total supply chain eventually in addition to satisfying service level requirements are incorporated in Supply chain management. The manufacturer who is in charge of the acquirement of raw materials, conversion into end products and distribution of the same to customers, is considered to be the manager of the supply chain. A huge problem that a majority of the supply chains aiming to reduce the supply chains costs besides improving customer service levels faces is that of the administration of the dynamic demand ${ }^{[9]}$. A variety of processes that aid the planning, implementation, control, manufacturing and the delivery processes originating from the raw material reserve to the point of utilization of the end product ${ }^{[6]}$, are considered to be an integral part of the supply chain managements. Shorter product lifecycles that lead to higher demand uncertainty and their effect on global markets accordingly increasing the supply chain complexity, results in severe problems in the management of the supply chain ${ }^{[8,10]}$. The research proposed tends to address four problems from the operational perspective: Inventory management and control; production, planning and scheduling; information sharing, coordination, monitoring and operation tools ${ }^{[9]}$.

The effective management of the supply chain has become unavoidable these days due to the firm increase in customer service levels ${ }^{[5]}$. The supply chain cost was immensely influenced by the overload or shortage of inventories. Thus inventory optimization has transpired into one of the most recent topics as far as supply chain management is considered ${ }^{[3,11,12]}$.

Inventory is defined as the ensemble of items stored by an enterprise for future use and a set of procedures called inventory systems aid in examination and control of the inventory ${ }^{[4]}$. It is possible to stock the inventory through various stages along the production and distribution supply chain ${ }^{[3]}$. The inventory system supports the estimation of amount of each item to be stored, when the low items should be restocked and the number of items that must be ordered or manufactured as soon as restocking becomes essential ${ }^{[4]}$. It is the responsibility of a supply manager to make a decision on the offers that need to be accepted in addition to updating the anticipated future inventory replacement costs.

Inventory optimization application organizes the latest techniques and technologies, thereby assisting the enhancement of inventory control and its management across an extended supply network. Some of the design objectives of inventory optimization are to optimize inventory strategies, thereby enhancing customer service, reducing lead times and costs and meeting market demand ${ }^{[3,11,12]}$. The design and management of the storage policies and procedures for raw materials, work-in-process inventories and typically, final products $^{[13]}$ are illustrated by the inventory control. The costs and lead times can be reduced and the responsiveness to the changing customer demands can be significantly improved and subsequently inventory can be optimized by the effective handling of the supply chain ${ }^{[8]}$.

The inventory and supply chain managers are mainly concerned about the estimation of the exact amount of inventory at each point in the supply chain free of excesses and shortages although the total supply chain cost is minimized. Owing to the fact that shortage of inventory yields to lost sales, whereas excess of inventory may result in pointless storage costs, the precise estimation of optimal inventory is indispensable ${ }^{[14]}$. In other words, there is a cost involved in manufacturing any product in the factory as well as in holding any product in the distribution center and agent shop. More the products manufactured or held, higher will be the holding cost. Meanwhile, there is possibility for the shortage of products. For the shortage of each product there will be a shortage cost. Holding excess stock levels as well as the occurrence of shortage for products lead to the increase in the supply chain cost.

This study supplements the previous study that focuses only on a single product ${ }^{[27]}$. In this study, we are considering the situation of multiple products and multiple members of the supply chain. Thus the complexity of the problem has been increased. We have developed a novel and efficient approach using Genetic Algorithm to solve this complexity. In order to minimize the total supply chain cost, the proposed approach clearly determines the most probable excess stock level and shortage level that are required for inventory optimization in the supply chain. In practice, the dynamic nature of the excess stock level and shortage level over all the periods is the typical problem occurring in inventory management. The determination of the stock level that occurs in a maximum rate is the vital operation to be performed. Thus, the maximum 
occurrences of stock level should be considered in order to optimize effectively. The employed fitness function of the genetic algorithm is formulated in such a way that it will consider the past periods to determine the necessary stock level. The proposed approach of genetic algorithm predicts the optimum stock levels of the future by considering the stock levels of the past years such that the total supply chain cost will be maintained as minimum.

Related works: A fresh Genetic Algorithm (GA) approach for the Integrated Inventory Distribution Problem (IIDP) has been projected by Abdel et al. ${ }^{[2]}$. They have developed a genetic representation and have utilized a randomized version of a formerly developed construction heuristic in order to produce the initial random population.

Pupong et al. ${ }^{[8]}$ have put forth an optimization tool that works on basis of a Multi-matrix Real-coded Generic Algorithm (MRGA) and aids in reduction of total costs associated with in supply chain logistics. They have incorporated procedures that ensure feasible solutions such as the chromosome initialization procedure, crossover and mutation operations. They have evaluated the algorithm with the aid of three sizes of benchmarking dataset of logistic chain network that are conventionally faced by most global manufacturing companies.

A technique to utilize in supply-chain management that supports the decision-making process for purchases of direct goods has been projected by ${ }^{[14]}$. RFQs have been constructed on basis of the projections for future prices and demand and the quotes that optimize the level of inventory each day besides minimizing the cost have been accepted. The problem was represented as a Markov Decision Process (MDP) that allows for the calculation of the utility of actions to be based on the utilities of substantial future states. The optimal quote requests and accepts at each state in the MDP were determined with the aid of Dynamic programming.

A supply chain management agent comprising of predictive, optimizing and adaptive components called the TacTex-06 has been put forth by ${ }^{[6]}$. TacTex-06 functions by making predictions regarding the future of the economy, such as the prices that will be proffered by component suppliers and the degree of customer demand and then strategizing its future actions so as to ensure maximum profit.

Beamon et $a l^{[13]}$ have presented a study on evaluations of the performance measures employed in supply chain models and have also displayed a framework for the beneficial selection of performance measurement systems for manufacturing supply chains.
Three kinds of performance measures have been recognized as mandatory constituents in any supply chain performance measurement system. New flexibility measures have also been created for the supply chains.

The accomplishment of beam-ACO in supply-chain management has been proposed by ${ }^{[22]}$. Beam-ACO has been used to optimize the supplying and logistic agents of a supply chain. A standard ACO algorithm has aided in the optimization of the distributed system. The application of Beam-ACO has enhanced the local and global results of the supply chain.

A beneficial industry case applying Genetic Algorithms (GA) has been proposed by ${ }^{[23]}$. The case has made use of GAs for the optimization of the total cost of a multiple sourcing supply chain system. The system has been exemplified by a multiple sourcing model with stochastic demand. A mathematical model has been implemented to portray the stochastic inventory with the many to many demand and transportation parameters as well as price uncertainty factors

A genetic algorithm which has been approved by $\mathrm{Lo}^{[24]}$ deals with the production-inventory problem with backlog in the real situations, with time-varied demand and imperfect production due to the defects in production disruption with exponential distribution. Besides optimizing the number of production cycles to generate a $(\mathrm{R}, \mathrm{Q})$ inventory policy, an aggregative production plan can also be produced to minimize the total inventory cost on the basis of reproduction interval searching in a given time horizon.

Barlas et al. ${ }^{[25]}$ have developed a System Dynamics simulation model of a typical retail supply chain. The intent of their simulation exercise was to build up inventory policies that enhance the retailer's revenue and reduce costs at the same instant. Besides, the research was also intended towards studying the implications of different diversification strategies

A supply chain model functioning under periodic review base-stock inventory system to assist the manufacturing managers at HP to administer material in their supply chains has been introduced by Lee et al. ${ }^{[26]}$. The inventory levels across supply chain members were obtained with the aid of a search routine.

Genetic algorithm: Genetic algorithm is a randomized search methodology having its roots in the natural selection process. Initially the neighborhood search operators (crossover and mutation) are applied to the preliminary set of solutions to acquire generation of new solutions. Solutions are chosen randomly from the existing set of solutions where the selection probability and the solution's objective function value are proportional to each other and eventually the aforesaid 
operators are applied on the chosen solutions. Genetic algorithms have aided in the successful implementation of solutions for a wide variety of combinatorial problems.

The robustness of the Genetic algorithms as search techniques have been theoretically and empirically proved $^{[17]}$. The artificial individual is the basic element of a GA. An artificial individual consists of a chromosome and a fitness value, similar to a natural individual. The individual's likelihood for survival and mating is determined by the fitness function ${ }^{[15]}$. In accordance with the Darwin's principle, individuals superior to their competitors, are more likely to promote their genes to the next generations. In accordance with this concept, in Genetic Algorithms, we encode a set of parameters mapped into a potential solution, named chromosome, to the optimization problem ${ }^{[16]}$. The population of candidate solutions is obtained through the process of selection, recombination and mutation performed in an iterative manner ${ }^{[18]}$.

Chromosomes refer to the random population of encoded candidate solutions with which the Genetic algorithms initiate with $^{[17]}$. Then the set (called a population) of possible solutions (called chromosomes) are generated $^{[21]}$. A function assigns a degree of fitness to each chromosome in every generation in order to use the best individual during the evolutionary process ${ }^{[20]}$. In accordance to the objective, the fitness function evaluates the individuals ${ }^{[18]}$. Each chromosome is evaluated using a fitness function and a fitness value is assigned. Then, three different operators-selection, crossover and mutation-are applied to update the population. A generation refers to an iteration of these three operators $^{[19]}$. The promising areas of the search space are focused in the selection step. The selection process typically keeps solutions with high fitness values in the population and rejects individuals of low quality ${ }^{[18]}$. Hence, this provides a means for the chromosomes with better fitness to form the Mating Pool (MP) ${ }^{[21]}$.

After the process of Selection, the crossover is performed. In the crossover operation, two new children are formed by exchanging the genetic information between two parent chromosomes (say $\mathrm{C} 1$ and $\mathrm{C} 2$ which are selected from the selection process) ${ }^{[22]}$. A crossover point is chosen at random by the crossover operator. At this point, two parent chromosomes break and then exchange the chromosome parts after that point. Consequently, the partial features of two chromosomes are combined to generate two off springs. The chromosome cloning takes place when a pair of chromosomes does not cross over, thus creating off springs that are exact copies of each parent ${ }^{[20]}$. The ultimate step in each generation is the mutation of individuals through the alteration of parts of their genes $^{[18]}$. Mutation alters a portion of a chromosome and thus institutes variability into the population of the subsequent generation ${ }^{[21]}$. Mutation, a rarity in nature, denotes the alteration in the gene and assists us in avoiding loss of genetic diversity ${ }^{[18]}$. Its chief intent is to ensure that the search algorithm is not bound on a local optimum $^{[20]}$.

\section{MATERIALS AND METHODS}

The inventory control for more number of products along with different levels of supply chain is a complex task.

Inventory optimization analysis using GA: To make the inventory control effective, the most primary objective is to predict where, why and how much of the control is required. Such a prediction is to be made here through the methodology we have proposed. To accomplish the same we are using Genetic algorithm and the optimal product that needs to be kept in the level of control is determined on the basis of the knowledge of the past records. This leads to an easy estimation of the level of stocks of the particular products to be maintained in the upcoming periods. For instance, we are taking a three stage supply chain having seven members and it is shown in Fig. 1.

As shown in Fig. 1, a factory is the parent of the chain and it is having two distribution centers Distribution center 1 and Distribution center 2. Each Distribution center further comprises of several agents but as stated in our exemplary case, each Distribution center is having two agents. So, in aggregate there are four agents, Agent1 and Agent2 for Distribution center 1 and Agent 3 and Agent 4 for Distribution center 2. The factory manufactures different products that would be supplied to the distribution centers. From the distribution center, the stocks will be moved to the corresponding agents. Our methodology is taking the responsibility here in determining the exact product that needs to be concentrated on and the amount of stock levels of the product to be maintained by the different members of the supply chain. Also, the methodology analyses whether the stock level of the particular product needs to be in abundance in order to avoid shortage of the product or needs to be held minimal in order to minimize the holding cost.

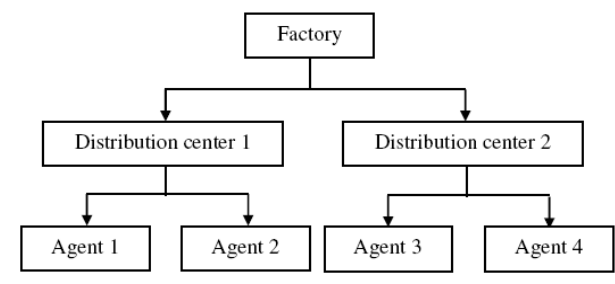

Fig. 1: 3 stage-7 member supply chain 
Table 1: The dataset for the analysis taken from the past periods

\begin{tabular}{|c|c|c|c|c|c|c|c|}
\hline Products & Factory & $\begin{array}{l}\text { Distribution } \\
\text { center } 1\end{array}$ & $\begin{array}{l}\text { Distribution } \\
\text { center } 2\end{array}$ & Agent 1 & Agent 2 & Agent 3 & Agent 4 \\
\hline P1 & 2000 & 500 & -300 & 30 & 120 & -130 & 200 \\
\hline P3 & -4000 & 200 & 400 & 200 & -300 & -450 & -160 \\
\hline P6 & 3000 & -700 & -600 & -450 & 80 & 230 & 400 \\
\hline$\vdots$ & $\vdots$ & $\vdots$ & $\vdots$ & $\vdots$ & $\vdots$ & $\vdots$ & $\vdots$ \\
\hline
\end{tabular}

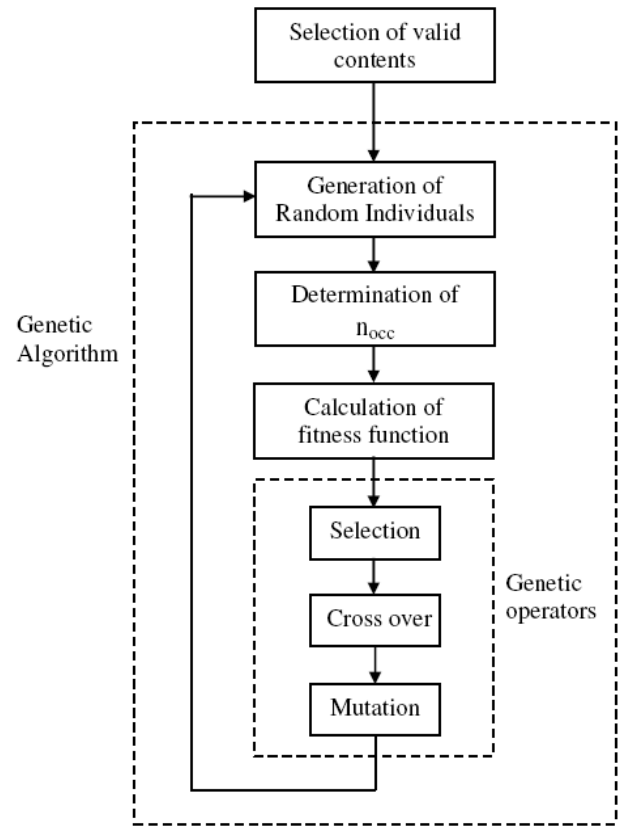

Fig. 2: Genetic Algorithm flow for the proposed inventory management analysis

The methodology flow as shown in Fig. 2 would analyze the past records very effectively and thus facilitate efficient inventory management with the contribution of Genetic Algorithm.

The analysis flow is initiated by the selection of valid records. The validation of records are done over the records of past periods, for instance, we consider a record of ten years, from August 1997 to July 2007 as past year's record set. The stock levels at the different supply chain members are held for different products, namely P1, P2, P3, P4, P5, P6 and P7. If seven products have been considered for the analysis, the stock levels for the seven products at each member of the chain throughout the ten years period are considered as data set as shown in the Table 1 . In the valid record set selection, records having nil values are neglected and the records having positive or negative values are selected for the analysis. This can be done by means of clustering algorithms, extraction algorithms or by any of the data mining functions. Hence the extraction function results in data sets having either positive or negative values.

\begin{tabular}{|l|c|c|c|c|c|c|c|}
\hline P4 & 6000 & -100 & -500 & 700 & -300 & 600 & -700 \\
\hline P1 & 2000 & 200 & -400 & 600 & -800 & -300 & 600 \\
\hline P5 & 5000 & 300 & -600 & 400 & 900 & -800 & -600 \\
\hline
\end{tabular}

Fig. 3: Random individual generated for the genetic operation

The record set having positive values represents excess stock levels of a particular product and the negative values represent shortage level of the particular product. This will be carried out for each product and for every member of the chain. Then the data set is subjected to Genetic Algorithm and the various steps performed in the genetic algorithm dedicated for our objective work are discussed below.

Generation of individuals: Each individual which is constituted by genes is generated with random values. Here, the chromosome of seven genes where the random values occupy each gene is generated along with the product representation. A random individual generated for the genetic operation is shown in the Fig. 3 .

Each gene of the chromosome displayed in the Fig. 3 is the stock level of the product with that particular member. As for our three individuals, Individual1 deals with product 1 , Individual 2 deals with product 4 and Individual 3 deals with product 5 . So, the first individual P1 represents 2000 excess stocks in factory, 200 excess stocks in distribution center 1, shortage of 400 stocks in Distribution center 2 and so on. In such a manner the other individuals represent the product $\mathrm{P} 4$ and the product $\mathrm{P} 5$.

After the generation of the individuals, the number of occurrences of the individual in the past records is determined. This is performed by the function count() and the total number of occurrences of that individual for the particular product is determined. This is equivalent to the number of occurrences of such situation of stock levels for the particular product in all the members throughout the period of ten years under consideration.

Evaluation of fitness function: A specific kind of objective function that enumerates the optimality of a 
solution in a genetic algorithm in order to rank certain chromosome against all the other chromosomes is known as Fitness function. Optimal chromosomes, or at least chromosomes which are near optimal, are permitted to breed and merge their datasets through one of the several techniques available in order to produce a new generation that will be better than the ones considered thus far.

The fitness function is given by:

$$
f(i)=\log \left(1-\frac{n_{\text {occ }}(i)}{n_{\text {tot }}}\right), i=1,2,3, \cdots, n
$$

Where:

$\mathrm{n}_{\text {occ }}(\mathrm{i})=$ The number of occurrences of the chromosome $\mathrm{i}$ in the record set

$\mathrm{n}_{\mathrm{tot}}=$ The total number of records that have been collected from the past or total number of data present in the record set

The fitness function mentioned ranks the randomly generated chromosome. Then, the chromosomes are subjected to the genetic operations.

Genetic operations: Once fitness calculation is done, Genetic operations are performed. Selection, Crossover and mutation comprise Genetic operations.

Selection: The selection operation is the initial genetic operation which is responsible for the selection of the fittest chromosome for further genetic operations.

This is done by offering ranks based on the calculated fitness to each of the prevailing chromosome. On the basis of this ranking, best chromosomes are selected for further proceedings.

Crossover: Among the numerous crossover operators in practice, for our complex operation, we have chosen two point crossover. From the matting pool, two chromosomes are subjected for the two point crossover. The crossover operation performed in our analysis is pictured in Fig. 4.

As soon as the crossover operation is completed, the genes of the two chromosomes present within the two crossover points get interchanged. The genes before the crossover point $\mathrm{C} 1$ and the genes beyond the crossover point $\mathrm{C} 2$ remain unaltered even after the crossover operation.

Mutation: The crossover operation is succeeded by the final stage of genetic operation known as Mutation. In the mutation, a new chromosome is obtained. This chromosome is totally new from the parent chromosome.

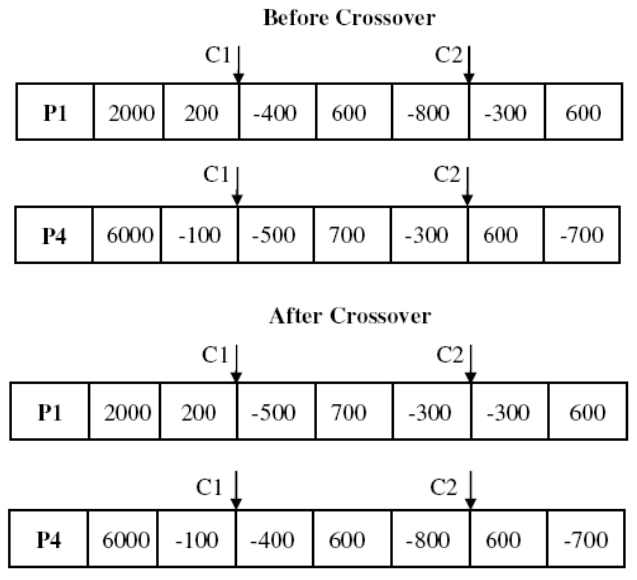

Fig. 4: Chromosomes are subjected for two point crossover operation

Before Mutation

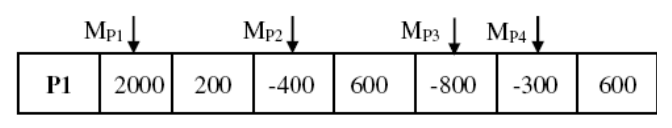

After Mutation

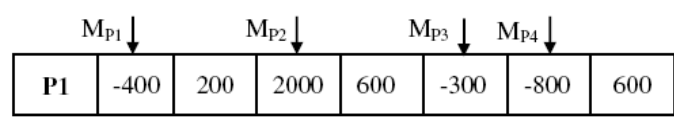

Fig. 5: Chromosome subjected for mutation operation

The concept behind this is the child chromosome thus obtained will be fitter than the parent chromosome. The performance of mutation operation is shown in Fig. 5.

As in Fig. 5 we have chosen four mutation points Mp1, Mp2, Mp3 and Mp4. The mutation is done on the particular gene present at the Mutation points. This pointing of gene is done randomly. Hence, the four mutation points may point any of the seven genes.

The process explained so far will be repeated along with the new chromosome obtained from the previous process. In other words, at the end of each of the iteration, a best chromosome will be obtained. This will be included with the chromosomes for the next iteration. Eventually, we obtain an individual which is the optimal one among all the possible individuals. Thus obtained chromosome has the optimal information about stock levels of the corresponding members of a particular product. From the information it can be concluded that the particular product and its corresponding stock levels play a significant role in the increase of supply chain cost. By controlling the stock level of that particular product in the upcoming periods, the supply chain cost can be minimized. 
Table 2: A sample data set constituted by the product Identification along with its stock levels in each member of the supply chain

\begin{tabular}{lrrrrrrr}
\hline PI & \multicolumn{1}{c}{ F1 } & \multicolumn{1}{c}{ F2 } & \multicolumn{1}{c}{ F3 } & \multicolumn{1}{c}{ F4 } & \multicolumn{1}{c}{ F5 } & \multicolumn{1}{c}{ F6 } & \multicolumn{1}{c}{ F7 } \\
\hline 5 & -407 & 379 & -981 & -864 & -391 & 999 & -196 \\
2 & -146 & -604 & 443 & 746 & -561 & -734 & 445 \\
4 & -962 & -524 & -685 & -254 & 205 & 446 & -469 \\
3 & -834 & 266 & 969 & 965 & 735 & 244 & -752 \\
3 & -449 & -282 & 577 & -926 & -414 & -200 & -743 \\
4 & 540 & -830 & -835 & 882 & -379 & 768 & -635 \\
4 & -371 & -736 & -299 & 634 & 448 & 756 & 340 \\
3 & -778 & -313 & 629 & -690 & 824 & -927 & 850 \\
2 & 351 & 293 & 328 & -732 & 357 & -566 & 685 \\
1 & 500 & 108 & 490 & -345 & -236 & 108 & -931 \\
5 & 844 & -728 & 286 & 740 & 686 & -421 & 424 \\
4 & -321 & 902 & -450 & -260 & -144 & 162 & 238 \\
3 & 775 & -394 & -520 & -792 & -927 & -879 & -507 \\
4 & 794 & 932 & -584 & 307 & -171 & -529 & -503 \\
2 & -122 & -686 & -620 & 424 & -891 & -824 & 941 \\
3 & 235 & 464 & 401 & 108 & 346 & 840 & -934 \\
5 & 218 & -848 & 836 & 133 & -554 & -939 & -834 \\
4 & 489 & 409 & 148 & 850 & 196 & 851 & -495 \\
3 & -422 & 638 & 676 & -112 & 539 & 107 & -440 \\
5 & 893 & 520 & -423 & -736 & -778 & 863 & -335 \\
\hline
\end{tabular}

\section{RESULTS}

The approach we have suggested for the optimization of inventory level and thereby efficient supply chain management has been implemented in the platform of MATLAB (MATLAB 7.4). The database consists of the records of stock levels held by each member of the supply chain for every period. In our implementation we have utilized five different products and these products are in circulation in the seven member supply chain network we have considered. The sample database which consists of the past records is shown in Table 2.

In the database we have tabulated in Table 1, the first field comprises of the product Identification (PI) and the other fields are related with the stock levels that were held by the respective seven members of the supply chain network. For example, the first attribute and first field of the database is ' 5 ' which refers the Product I.D. '5'. The corresponding fields of the same attribute denote the stock levels of the product I.D. ' 5 ' in the respective members of the supply chain. Similarly, different sets of stock levels are held by the database.

\section{DISCUSSION}

As per the proposed analysis based on GA, we have generated the random initial chromosome as '[ $\left[\begin{array}{llllllll}4 & 895 & -732 & 485 & -213 & -270 & 314 & -850\end{array}\right]$ '. This will represent the database content as shown in the Fig. 6.

\begin{tabular}{|c|c|c|c|c|c|c|c|}
\hline PI & F1 & F2 & F3 & F4 & F5 & F6 & F7 \\
\hline 4 & 895 & -732 & 485 & -213 & -270 & 314 & -850 \\
\hline
\end{tabular}

Fig. 6: Random inventory generated initially for the GA based analysis

\begin{tabular}{|c|c|c|c|c|c|c|c|}
\hline PI & F1 & F2 & F3 & F4 & F5 & F6 & F7 \\
\hline 1 & 697 & -906 & 304 & 257 & 849 & -444 & -845 \\
\hline
\end{tabular}

Fig. 7: The final best chromosome obtained from the analysis

Figure 6 clearly describes that the first element of the chromosome generated randomly points to the product ID of the database. Similarly, all the other elements of the generated chromosome point to the corresponding fields of the database. In this manner two different random chromosomes have been generated and they will be subjected for genetic operations like Fitness evaluation, Selection, Crossover and Mutation.

An iteration involving all these processes was carried out so as to obtain the best chromosome. Here for instance, we have chosen the iteration value as '100' and so hundred numbers of iterative steps will be performed. The best chromosome we have obtained as result is' $\left[\begin{array}{lllllllll}1 & 697 & -906 & 304 & 257 & 849 & -444 & -845\end{array}\right]^{\prime}$ and its database format is depicted in the Fig. 7.

The final chromosome we have obtained from the GA based analysis shown in the Fig. 7 is the inventory level that caused maximum increase of supply chain cost. By maintaining the inverse of these kinds of stock levels like '[ $\left.\begin{array}{llllllll}1 & -697 & 906 & -304 & -257 & -849 & 444 & 845\end{array}\right]$ ' we can minimize the supply chain cost. This is the optimal stock level that needs to be maintained in order to make the supply chain cost minimum. Thus by following the predicted stock levels, we can avoid the increase of supply chain cost.

\section{CONCLUSION}

Inventory management is an important component of supply chain management. We have proposed an innovative and efficient methodology that uses Genetic Algorithms to precisely determine the most probable excess stock level and shortage level required for inventory optimization in the supply chain such that the total supply chain cost is minimal. The proposed approach was implemented and its performance was evaluated using MATLAB 7.4. The performance of Genetic Algorithm was well as predicted. By following the proposed genetic algorithm based approach for inventory management, we have successfully obtained the optimized stock levels at all members of the supply 
chain. The complexity of increasing the number of products through the supply chain has also been resolved by the proposed approach. We determined the products due to which the members of the supply chain incurred extra holding or shortage cost. More specifically, the inventory is optimized in the whole supply chain regardless of the number of products and the number of members in the supply chain. The proposed approach of inventory management has achieved the objectives which are the minimization of total supply chain cost and the determination of the products due to which the supplier endured either additional holding cost or shortage cost.

\section{REFERENCES}

1. Sarmiento, A., L. Rabelo, R. Lakkoju and R. Moraga, 2007. Stability analysis of the supply chain by using neural networks and genetic algorithms. Proceedings of the Conference on Winter Simulation, Dec. 9-12, IEEE Xplore Press, Washington, DC., pp: 1968-1976. DOI: 10.1109/WSC.2007.4419826

2. Abdelmaguid, T.F. and M.M. Dessouky, 2006. A genetic algorithm approach to the integrated inventory-distribution problem. Int. J. Prod. Res., 44: 4445-4464. DOI: 10.1080/00207540600597138

3. Golden Embryo Technologies Pvt. Ltd., 2004. Optimization Engine for Inventory Control. White paper, Maharastra, India. www.goldenembryo.net/downloads/inventory_cont rol.pdf

4. Levi, R., M. Pal, R.O. Roundy and D.B. Shmoys, 2007. Approximation algorithms for stochastic inventory control models. Math. Operat. Res., 32: 284-302. DOI: 10.1287/moor.1060.0205

5. Peter, M. and N. Karoly, 2006. A new inventory control method for supply chain management. Proceeding of the 12th International Conference on Machine Design and Production, Sep. 5-8, Kusadasi, $\quad$ Turkey, $\quad$ pp: 1-16. http://citeseerx.ist.psu.edu/viewdoc/download;jsess ionid=BA3220BFF2B74E13BE72ECAD547487E D?doi=10.1.1.98.3739\&rep=rep1\&type $=$ pdf

6. Pardoe, D. and P. Stone, 2007. An Autonomous Agent for Supply Chain Management. In: Handbooks in Information Systems Series: Business Computing, Adomavicius, G. and A. Gupta (Eds.). Elsevier. Amsterdam. http://www.cs.utexas.edu/ pstone/Papers/bib2html/ b2hd-TacTex-Book07.html
7. Pongcharoen, P., A. Khadwilard and A. Klakankhai, 2007. Multi-matrix real-coded genetic algorithm for minimising total costs in logistics chain network. Int. J. Intell. Syst. Technol., 3: 1-6. http://www.waset.org/ijist/v3/v3-1-1.pdf

8. Ganeshan, R., E. Jack, M.J. Magazine and P. Stephens, 1999. A Taxonomic Review of Supply Chain Management Research. Quantitative Models for Supply Chain Management. 6th Edn., Kluwer Academic Publishers, Massachusetts, ISBN: 0792383443, pp: 885.

9. Rajesh Gangadharan, 2007. Supply Chain Strategies to Manage Volatile Demand. http://www.supplychainbrain.com/content/index.php?id= 374\&no_cache=1\&tx_ttnews\%5Btt_news\%5D=2207

10. Joines, J.A., D. Gupta, M.A. Gokce, R.E. King and M.G. Kay, 2002. Supply chain multi-objective simulation optimization. Proceedings of IEEE Winter Simulation Conference, Dec. 8-11, Raleigh, USA., $\quad$ pp: 1306-1314. DOI: 10.1109/WSC.2002.1166395

11. Liu, J., H. Gao and J. Wang, 2000. Air material inventory optimization model based on genetic algorithm. Proceedings of the 3rd World Congress on Intelligent Control and Automation, June 28July 2, Hefei, China, pp: 1903-1904. DOI: 10.1109/WCICA.2000.862809

12. Adams, C.M., 2004. Inventory optimization techniques, system vs. item level inventory analysis. Proceeding of the Annual Symposium RAMS Reliability and Maintainability. Jan. 26-29, pp: 55-60. DOI: 10.1109/RAMS.2004.1285423.

13. Beamon, B.M., 1998. Supply chain design and analysis: Models and methods. Int. J. Prod. Econ., 55: 281-294.

http://cat.inist.fr/?aModele=afficheN\&cpsidt=1700230

14. Buffett, S. and N. Scott, 2004. An algorithm for procurement in supply chain management. Proceeding of the 3rd International Conference on Autonomous Agents and Multi-Agent Systems, July 20, New York, USA., pp: 9-14. http://iititi.nrc-cnrc.gc.ca/iit-publications-iti/docs/NRC47455.pdf

15. Khadwilard, A. and P. Pongcharoen, 2007. Application of genetic algorithm for trajectory planning of two degrees of freedom robot arm with two dimensions. Thammasat. Int. J. Sci. Technol., 12: 88-94.

http://www.tijsat.tu.ac.th/issues/2007/no2/2007_V1 2_No2_8.PDF

16. Sharbafi, M.A., M.S. Herfeh, C. Lucas and A.M. Nejad, 2006. An innovative fuzzy decision making based genetic algorithm. Proc. World Acad. Sci. Eng. Technol., 13: 180-183. http://www.waset.org/pwaset/v13/v13-36.pdf 
17. Behzadi, S., A.A. Alesheikh and E. Poorazizi, 2008. Developing a genetic algorithm to solve shortest path problem on a raster data model. Proc. J. Applied Sci., 8: 3289-3293. DOI: 10.3923/jas.2008.3289.3293

18. Butter, T., F. Rothlauf, J. Grahl, T. Hildenbrand and J. Arndt, 2006. Developing genetic algorithms and mixed integer linear programs for finding optimal strategies for a student's "sports" activity. Proceeding of the Working Papers in Information Systems, Mar. 2006, University of Mannheim, pp: 1-16. http://wifo1.bwl.unimannheim.de/fileadmin/files/publications/working paper.pdf

19. Soryani, M. and N. Rafat, 2006. Application of genetic algorithms to feature subset selection in a farsi OCR. Proc. World Acad. Sci. Eng. Technol., 13: 113-116.

http://www.waset.org/pwaset/v18/v18-22.pdf

20. Tareeq, S.M., R. Parveen, L.J. Rozario and Md. Al-Amin Bhuiyan, 2007. Robust face detection using genetic algorithm. Inform. Technol. J., 6: 142-147. DOI: 10.3923/itj.2007.142.147

21. Qureshi, S.A., S.M. Mirza and M. Arif, 2006. Fitness function evaluation for image reconstruction using binary genetic algorithm for parallel ray transmission tomography. Proceeding of the International Conference on Emerging Technologies, Nov. 13-14, Peshawar, pp: 196-201. DOI: 10.1109/ICET.2006.336012
22. Caldeira, J.L., R.C. Azevedo, C.A. Silva and J.M.C. Sousa, 2007. Supply-chain management using ACO and beam-ACO algorithms. Proceeding of the IEEE International Fuzzy Systems Conference, July 23-26, London, pp: 1-6. DOI: 10.1109/FUZZY.2007.4295615

23. Wang, K. and Y. Wang, 2008. Applying genetic algorithms to optimize the cost of multiple sourcing supply chain systems-An industry case study. Stud. Comput. Intell., 92: 355-372. DOI: 10.1007/978-3-540-76286-7_16

24. Lo, C.Y., 2008. Advance of dynamic productioninventory strategy for multiple policies using genetic algorithm. Inform. Technol. J., 7: 647-653. DOI: 10.3923/itj.2008.647.653

25. Yaman, B. and A. Aksogan, 1996. Product diversification and quick response order strategies in supply chain management. Proceedings of the 14th International Conference of the System Dynamics Society, Cambridge, USA.

http://www.systemdynamics.org/conferences/1996/ proceed/papers/barla051.pdf

26. Lee, H.L. and C. Billington, 1995. The evolution of supply-chain-management models and practice at Hewlett-Packard. Interface, 25: 42-63. DOI: 10.1287/inte.25.5.42

27. Radhakrishnan, P., V.M. Prasad and M.R. Gopalan, 2009. Inventory optimization in supply chain management using genetic algorithm. Int. J. Comput. Sci. Network Secur., 9: 1-8. http://paper.ijcsns.org/07_book/200901/20090105.pdf 